\title{
Strong correlation and charge localisation in Kohn-Sham theories with fractional orbital occupations
}

\author{
Maria Hellgren*,† and Tim Gould \\ †Sorbonne Université, Muséum National d'Histoire Naturelle, UMR CNRS 7590, Institut \\ de Minéralogie, de Physique des Matériaux et de Cosmochimie (IMPMC), 4 place Jussieu, \\ 75005 Paris, France \\ $\ddagger$ Qld Micro- and Nanotechnology Centre, Griffith University, Nathan, Qld 4111, Australia \\ E-mail: maria.hellgren@sorbonne-universite.fr
}

\begin{abstract}
We study static correlation and delocalisation errors and show that even methods with good energies can yield significant delocalization errors that affect the density, leading to large errors in predicting e.g. dipole moments. We illustrate this point by comparing existing state-of-art approaches with an accurate exchange correlation functional based on a generalised valence-bond ansatz, in which orbitals and fractional occupations are treated as variational parameters via an optimized effective potential (OEP). We show that the OEP exhibits step and peak features which, similar to the exact Kohn-Sham (KS) potential of DFT, are crucial to prevent charge delocalization. We further show that the step is missing in common approximations within reduced density matrix functional theory resulting in delocalization errors comparable to those found in DFT approximations. Finally, we explain the delocalization error as coming
\end{abstract}


from an artificial mixing of the ground state with a charge-transfer excited state which is avoided if occupation numbers exhibit discontinuities.

\section{Introduction}

Electron delocalisation errors and related issues ${ }^{1-4}$ have been the subject of intensive study in recent years, due to their importance to molecular modeling, particularly in charge transfer systems. Delocalisation errors arise in most common ab initio approximations; ${ }^{5-7}$ within density functional theory (DFT) but also in more advanced many-body methods such as MP2, GW and beyond. ${ }^{8-11}$ These errors are most prominent when breaking molecular bonds, and are responsible for the dramatic failures to correctly predict the charge distribution at dissociation. $^{12-14}$

In addition to delocalization errors much attention has been paid to overcoming the static correlation error in density functional approaches. ${ }^{15-24}$ This error is manifested in the strongly overestimated dissociation energy of molecules composed of open-shell atoms. Very often static correlation and delocalisation errors occur simultaneously, exemplified by the LiH molecule. Consequently, such cases are extremely difficult for most density functional approximations, which reproduce neither correct densities nor energetics.

An alternative pathway is to use reduced density matrix functional theory (RDMFT), by directly approximating the first- and second-order reduced density matrices. ${ }^{1,25,26}$ The resulting energy expression then becomes a simultaneous functional of (natural) orbital occupation factors, and a corresponding set of orthonormal (natural) orbitals. With an appropriate choice of RDMFT approximation, strong static correlation effects can then be directly accommodated within a (natural) orbital framework.

It is also possible to accommodate orbitals within a DFT framework, by employing an optimized effective potential (OEP) approach ${ }^{27,28}$ together with an appropriate orbital functional. In so doing, one hopes that an appropriate choice of functional can lead to an 
approach that intrinsically avoids delocalization errors. The best known OEP approach is the exact-exchange (EXX) approximation within DFT. In EXX, one looks for the Hartree-Fock energy of a system using orbital functions $\varphi_{i}$ satisfying a common, local Hamiltonian

$$
\hat{h} \varphi_{i}(\mathbf{r})=\left[\hat{T}+V_{\operatorname{EXX}}(\mathbf{r})\right] \varphi_{i}(\mathbf{r})=\epsilon_{i} \varphi_{i}(\mathbf{r})
$$

EXX is known to reproduce many of the exact features ${ }^{29-33}$ of the true Kohn-Sham (KS) potential and, as such, largely resolves the delocalization problem in single-reference systems.

However, unless spin symmetry is allowed to break, the EXX approximation does a poor job of predicting the energy and density of dissociation into open-shell atoms, where the outermost electrons are strongly correlated. The majority of the error is caused by a failure to localize the outermost electrons on the different odd electron number atoms. Instead, the highest occupied molecular orbital (HOMO, H) is doubly occupied, with electrons shared across the two species. This allows "ghost interactions" to cause a static correlation error, with energy cost $1 / 2\left\langle\varphi_{\mathrm{H}}^{2}|v| \varphi_{\mathrm{H}}^{2}\right\rangle^{9}$ between opposite-spin electrons that should be excluded by the multi-determinant nature of the groundstate.

In this work we employ a multi-configurational approach that avoids ghost interactions and delocalisation errors via an optimized effective potential scheme. The energy is constructed from a wave function ansatz - built from one-electron orbitals defined as nonorthogonal linear combinations of KS orbitals. The KS orbitals are then optimized via an effective local potential. This scheme differs from other OEP schemes utilising unoccupied orbitals $^{9,34,35}$ in that the unoccupied orbitals are employed to remedy deficiencies of the reference system - similar to the local RDMFT approach ${ }^{36}$ or other multi-configurational OEP methods. ${ }^{37,38}$

We reveal the important role played by the effective potential in dealing with charge localization, even in theories which depend explicitly on occupation numbers, and how a failure to correctly predict the potential (and thus orbitals) leads to delocalisation errors 
even in methods that give, to leading order, correct correlation energies. This error manifests in measurable quantities, such as dipole moments.

\section{Optimized effective potential}

In this section we derive the OEP within theories that allow fractionally occupied KS orbitals. We employ a multiconfigurational approach similar to the ones proposed in Refs. 37,38 which we then, for two electrons, connect to the local RDMFT theory. ${ }^{36}$

\subsection{Wavefunction ansatz based on optimized Kohn-Sham orbitals}

The use of doubly occupied KS orbitals in DFT is an, in principle, exact construction. But, it is a bad starting point for treating strongly correlated electrons which are spatially localised and thus more naturally treated by localized orbitals. Spin-DFT can sometimes achieve spatial localisation; at the expense of breaking the fundamental spin symmetries. A proper treatment of correlation is provided by multi-configurational wavefunction approaches, but with the drawback that several configurations may be needed to converge the energy (or density).

In the following, we will explore the possibility of optimising the orbitals via a KS system with a local potential, common to all orbitals. As a proof-of-principle we propose a twoelectron model that captures challenging multi-reference physics, but is amenable to exact solutions of all properties. We can thus use it to illustrate important features of orbital-based approximations for multi-reference physics, in a highly controlled fashion.

We construct a generalized valence-bond-like ansatz ${ }^{39}$ of two Slater determinants built up from a set of nonorthogonal correlated orbitals that are allowed to localise via the HOMO 
and LUMO of the KS system

$$
\begin{aligned}
& \psi_{A}(\mathbf{r})=\cos \theta \varphi_{\mathrm{H}}(\mathbf{r})+\sin \theta \varphi_{\mathrm{L}}(\mathbf{r}) \\
& \psi_{B}(\mathbf{r})=\cos \theta \varphi_{\mathrm{H}}(\mathbf{r})-\sin \theta \varphi_{\mathrm{L}}(\mathbf{r}) .
\end{aligned}
$$

Unlike the KS orbitals, $\varphi_{\mathrm{H}}$ and $\varphi_{\mathrm{L}}$, the correlated orbitals are not eigenstates of an effective Hamiltonian. Only at $\theta=0$ do both $\psi_{A}$ and $\psi_{B}$ reduce to the KS HOMO orbital, and we recover double occupancy. As $\theta$ is increased, more of the LUMO orbital is mixed in and eventually at $\theta=\pi / 4, \psi_{A}$ and $\psi_{B}$ become orthogonal. The more the KS orbitals are delocalized, the more the correlated orbitals will be localized, and vice versa.

The two orbitals are then used to construct a singlet wavefunction

$$
|\Psi\rangle=\frac{1}{\sqrt{N}}\{|A \uparrow B \downarrow\rangle+|B \uparrow A \downarrow\rangle\}
$$

where $N=4\left(\cos ^{4} \theta+\sin ^{4} \theta\right)$ is the normalization constant. We notice that when $\theta=0$ this is just the Hartree-Fock (HF) wave function ansatz. We can also write (4) as

$$
|\Psi\rangle=\frac{1}{\sqrt{N}}\left[\psi_{A}(\mathbf{r}) \psi_{B}\left(\mathbf{r}^{\prime}\right)+\psi_{B}(\mathbf{r}) \psi_{A}\left(\mathbf{r}^{\prime}\right)\right]|S\rangle
$$

where $|S\rangle$ is the spin-singlet wavefunction.

Taking the expectation value of the full many-body Hamiltonian with respect to the wave function in Eq. (4) we find the density and kinetic energy to be

$$
\begin{aligned}
& n^{\theta}(\mathbf{r})=2 f_{\mathrm{H}}\left|\varphi_{\mathrm{H}}(\mathbf{r})\right|^{2}+2 f_{\mathrm{L}}\left|\varphi_{\mathrm{L}}(\mathbf{r})\right|^{2} \equiv\langle\Psi|\hat{n}(\mathbf{r})| \Psi\rangle \\
& T_{s}^{\theta}=2 f_{\mathrm{H}}\left\langle\varphi_{\mathrm{H}}|\hat{T}| \varphi_{\mathrm{H}}\right\rangle+2 f_{\mathrm{L}}\left\langle\varphi_{\mathrm{L}}|\hat{T}| \varphi_{\mathrm{L}}\right\rangle \equiv\langle\Psi|\hat{T}| \Psi\rangle .
\end{aligned}
$$

The occupation numbers $f_{H}=4 \cos ^{4} \theta / N$ and $f_{L}=4 \sin ^{4} \theta / N$ are related to the fourth 
power of the expansion coefficients of the correlated orbitals $\psi_{A}$ and $\psi_{B}$ and the factor of two comes from the spin-summation.

The electron-electron interaction is given by

$$
\begin{aligned}
E_{\mathrm{Hxc}}^{\theta}= & \frac{2}{N} \int d \mathbf{r} d \mathbf{r}^{\prime}\left|\psi_{A}(\mathbf{r})\right|^{2} v\left(\mathbf{r}, \mathbf{r}^{\prime}\right)\left|\psi_{B}\left(\mathbf{r}^{\prime}\right)\right|^{2} \\
& +\frac{2}{N} \int d \mathbf{r} d \mathbf{r}^{\prime} \psi_{A}(\mathbf{r}) \psi_{B}(\mathbf{r}) v\left(\mathbf{r}, \mathbf{r}^{\prime}\right) \psi_{A}\left(\mathbf{r}^{\prime}\right) \psi_{B}\left(\mathbf{r}^{\prime}\right) \\
= & \int d \mathbf{r} d \mathbf{r}^{\prime} n_{\mathrm{HL}}^{1 / 2}\left(\mathbf{r}, \mathbf{r}^{\prime}\right) v\left(\mathbf{r}, \mathbf{r}^{\prime}\right) n_{\mathrm{HL}}^{1 / 2}\left(\mathbf{r}^{\prime}, \mathbf{r}\right)
\end{aligned}
$$

where

$$
n_{\mathrm{HL}}^{1 / 2}\left(\mathbf{r}, \mathbf{r}^{\prime}\right)=\sqrt{f_{\mathrm{H}}} \varphi_{\mathrm{H}}(\mathbf{r}) \varphi_{\mathrm{H}}\left(\mathbf{r}^{\prime}\right)-\sqrt{f_{\mathrm{L}}} \varphi_{\mathrm{L}}(\mathbf{r}) \varphi_{\mathrm{L}}\left(\mathbf{r}^{\prime}\right) .
$$

We have called this term the Hartree and exchange and correlation (Hxc) energy but we note that it does not contain kinetic correlation contributions.

Finally, we use Eqs. (6)-(8) to generate the total energy

$$
E=\min _{\theta}\left[T_{s}^{\theta}+\int d \mathbf{r} V_{\mathrm{Ext}}(\mathbf{r}) n^{\theta}(\mathbf{r})+E_{\mathrm{Hxc}}^{\theta}\right],
$$

where the energy minimization over $\theta$ determines the amount of localization of $\psi_{A / B}$, via its optimizing value $\theta_{0}$. We henceforth implicitly define quantities using $\theta=\theta_{0}$ and drop the superscript $\theta$, e.g. $n \equiv n^{\theta_{0}}$.

The ansatz described above is known to capture $\mathrm{H}_{2}$ dissociation rather well if the orbitals are expressed in a Gaussian basis and coefficients are optimized variationally. The crucial difference in the approach presented here is that the orbitals are expressed in terms of the $\mathrm{HOMO} / \mathrm{LUMO} \mathrm{KS}$ orbitals that are optimised via an effective local KS potential. This potential is derived from the condition that the total energy at every fixed value of $\theta$ is 
stationary with respect to variations of the total potential $V_{s}(\mathbf{r})=V_{\mathrm{Ext}}(\mathbf{r})+V_{\mathrm{Hxc}}(\mathbf{r})$, i.e.,

$$
\frac{\delta E^{\theta}}{\delta V_{s}}=\frac{\delta T_{s}^{\theta}}{\delta V_{s}}+\int V_{\mathrm{Ext}} \frac{\delta n^{\theta}}{\delta V_{s}}+\frac{\delta E_{\mathrm{Hxc}}^{\theta}}{\delta V_{s}}=0 .
$$

This leads to an OEP type of equation for the Hxc potential

$$
\int d \mathbf{r}^{\prime} \frac{\delta n^{\theta}\left(\mathbf{r}^{\prime}\right)}{\delta V_{s}(\mathbf{r})} V_{\mathrm{Hxc}}\left(\mathbf{r}^{\prime}\right)=\frac{\delta E_{\mathrm{Hxc}}^{\theta}}{\delta V_{s}(\mathbf{r})}
$$

The variation of the density with respect to $V_{s}$ can be obtained from first order (static) perturbation theory

$$
\frac{\delta n^{\theta}\left(\mathbf{r}^{\prime}\right)}{\delta V_{s}(\mathbf{r})}=\sum_{i=H, L} \sum_{j \neq i} 4 f_{i} \frac{\varphi_{i}(\mathbf{r}) \varphi_{j}(\mathbf{r}) \varphi_{i}\left(\mathbf{r}^{\prime}\right) \varphi_{j}\left(\mathbf{r}^{\prime}\right)}{\varepsilon_{i}-\varepsilon_{j}}
$$

The variation of the Hxc energy is straightforwardly evaluated using Eq. (8), giving

$$
\begin{aligned}
\frac{\delta E_{\mathrm{Hxc}}^{\theta}}{\delta V_{s}(\mathbf{r})}= & \sum_{i=H, L} \sum_{j \neq i} 4 f_{i} \frac{\varphi_{i}(\mathbf{r}) \varphi_{j}(\mathbf{r})}{\varepsilon_{i}-\varepsilon_{j}} \int d \mathbf{r}^{\prime \prime} d \mathbf{r}^{\prime} \varphi_{i}\left(\mathbf{r}^{\prime \prime}\right) \varphi_{j}\left(\mathbf{r}^{\prime \prime}\right) v\left(\mathbf{r}^{\prime \prime}, \mathbf{r}^{\prime}\right)\left|\varphi_{i}\left(\mathbf{r}^{\prime}\right)\right|^{2} \\
& -\sum_{j \neq H} 4 \sqrt{f_{H}} \sqrt{f_{L}} \frac{\varphi_{H}(\mathbf{r}) \varphi_{j}(\mathbf{r})}{\varepsilon_{H}-\varepsilon_{j}} \int d \mathbf{r}^{\prime \prime} d \mathbf{r}^{\prime} \varphi_{H}\left(\mathbf{r}^{\prime \prime}\right) \varphi_{j}\left(\mathbf{r}^{\prime}\right) v\left(\mathbf{r}^{\prime \prime}, \mathbf{r}^{\prime}\right) \varphi_{L}\left(\mathbf{r}^{\prime}\right) \varphi_{L}\left(\mathbf{r}^{\prime \prime}\right) \\
& -\sum_{j \neq L} 4 \sqrt{f_{H}} \sqrt{f_{L}} \frac{\varphi_{L}(\mathbf{r}) \varphi_{j}(\mathbf{r})}{\varepsilon_{L}-\varepsilon_{j}} \int d \mathbf{r}^{\prime \prime} d \mathbf{r}^{\prime} \varphi_{L}\left(\mathbf{r}^{\prime \prime}\right) \varphi_{j}\left(\mathbf{r}^{\prime}\right) v\left(\mathbf{r}^{\prime \prime}, \mathbf{r}^{\prime}\right) \varphi_{H}\left(\mathbf{r}^{\prime}\right) \varphi_{H}\left(\mathbf{r}^{\prime \prime}\right) .
\end{aligned}
$$

The resulting OEP equation is very similar in structure to the exact-exchange OEP equation. Therefore, similar numerical techniques can be used to solve it. In this work we have expanded both orbitals and orbital products in a spline basis set. In this way, the OEP equation becomes a matrix equation, in which the potential is obtained by inverting the response matrix [Eq. (13)] in a subspace defined by fixing the HOMO energy to, e.g., the ionization energy. For more details regarding the OEP implementation see Refs. 40,41.

The main difference to previous work is that, here, the potential is evaluated at some starting value $\theta_{1}$ for which the corresponding total energy and orbitals are calculated. This 
procedure is repeated until the minimum energy at $\theta_{0}$ is found. Testing revealed that the two minimisations (over $\theta$ and the orbitals) can be carried out in either order. We optimise the orbitals first, and then the angle.

We note that the effective Hxc potential in Eq. (12) is different from the standard DFT Hxc potential with integer occupation numbers but serves a similar role. It is, in fact, equivalent to a potential generated through excited-state ensemble DFT ${ }^{42,43}$ and it thus inherits the usual good properties of KS potentials, such as uniqueness; and is fully amenable to the usual KS mechanics, such as division into kinetic, Hartree-exchange and correlation compo-

nents. ${ }^{44,45}$ Note, however, that within this formalism the division of Hartree, exchange and correlation terms into kinetic and electrostatic components differs slightly from conventional DFT. Their sum, nonetheless, remains the same.

Although we restrict ourselves to two-electron systems the combination of a wavefunction ansatz with orbitals optimised via a KS potential can be made more general. ${ }^{38}$ In this work our aim is to study the reliability and exact features of this local KS potential (from now on referred to as COEP [correlated orbital OEP]) in situations where density errors can be significant. We thus seek to reveal features of the exact potential that must be dealt with by approximations for more complex cases.

\subsection{Connection to reduced density matrix functional theory}

The reduced density matrix of the many-electron wavefunction is defined as

$$
n\left(\mathbf{x}, \mathbf{x}^{\prime}\right)=N \int d \mathbf{x}_{2-N} \Psi\left(\mathbf{x}, \mathbf{x}_{2} \ldots \mathbf{x}_{N}\right) \Psi^{*}\left(\mathbf{x}^{\prime}, \mathbf{x}_{2} \ldots \mathbf{x}_{N}\right)
$$

where the variable $\mathbf{x}$ carries both space and spin degrees of freedom. It is often written in terms of its spin eigenfunctions (natural orbitals) $\phi_{i}(\mathbf{x})$ and eigenvalues (occupation numbers) 
$f_{i}$

$$
n\left(\mathbf{x}, \mathbf{x}^{\prime}\right)=\sum_{i} f_{i} \phi_{i}(\mathbf{x}) \phi_{i}^{*}\left(\mathbf{x}^{\prime}\right) .
$$

It can be directly shown that the natural orbitals of the wavefunction in Eq. (5) are just the KS HOMO/LUMO orbitals, and the occupation numbers are given by the factors $f_{\mathrm{H} / \mathrm{L}}$ defined above. It is then easy to see that the derivation leading to the expression for the total energy Eqs. (7-8) is valid also for the exact wavefunction provided it is expressed in terms of its natural orbitals and occupation numbers. ${ }^{25}$ The COEP approximation defined in the previous section can thus alternatively be derived from the exact RDMFT energy expression with the restriction of keeping only two natural orbitals, further assumed to be generated by an effective local potential. The use of a local potential in RDMFT is always approximate but recent studies suggest that it does not impose a severe restriction. ${ }^{36}$ For this reason we refer to COEP as a "nearly exact" model, in the sense that it accurately captures strong correlations, and misses only a small fraction of dynamic correlations due to the restriction of keeping only two orbitals.

A common approximation to the exchange correlation energy in RDMFT is the Müller functional ${ }^{46,47}$ obtained by taking the square root of the occupation numbers in the Fock exchange term. It can thus be written as

$$
E_{\mathrm{xc}}^{\text {Müller }}=-\frac{1}{2} \int d \mathbf{x} d \mathbf{x}^{\prime} n^{1 / 2}\left(\mathbf{x}, \mathbf{x}^{\prime}\right) v\left(\mathbf{r}, \mathbf{r}^{\prime}\right) n^{1 / 2}\left(\mathbf{x}^{\prime}, \mathbf{x}\right)
$$

where $n^{1 / 2}\left(\mathbf{x}, \mathbf{x}^{\prime}\right)=\sum_{i} \sqrt{f_{i}} \phi_{i}(\mathbf{x}) \phi_{i}\left(\mathbf{x}^{\prime}\right)$. The corresponding local Müller potential at fixed occupations is determined by an OEP equation like Eq. (12) but with the xc part of the energy given by Eq. (17), and the Hartree part calculated in the conventional way. ${ }^{36}$

With some similarities to the nearly exact functional [Eq. (8)] the Müller functional captures static correlation rather well but tends to overestimate the correlation energy. In Ref. 48 it was shown that spurious magnetic instabilities occur in the Hubbard dimer and 

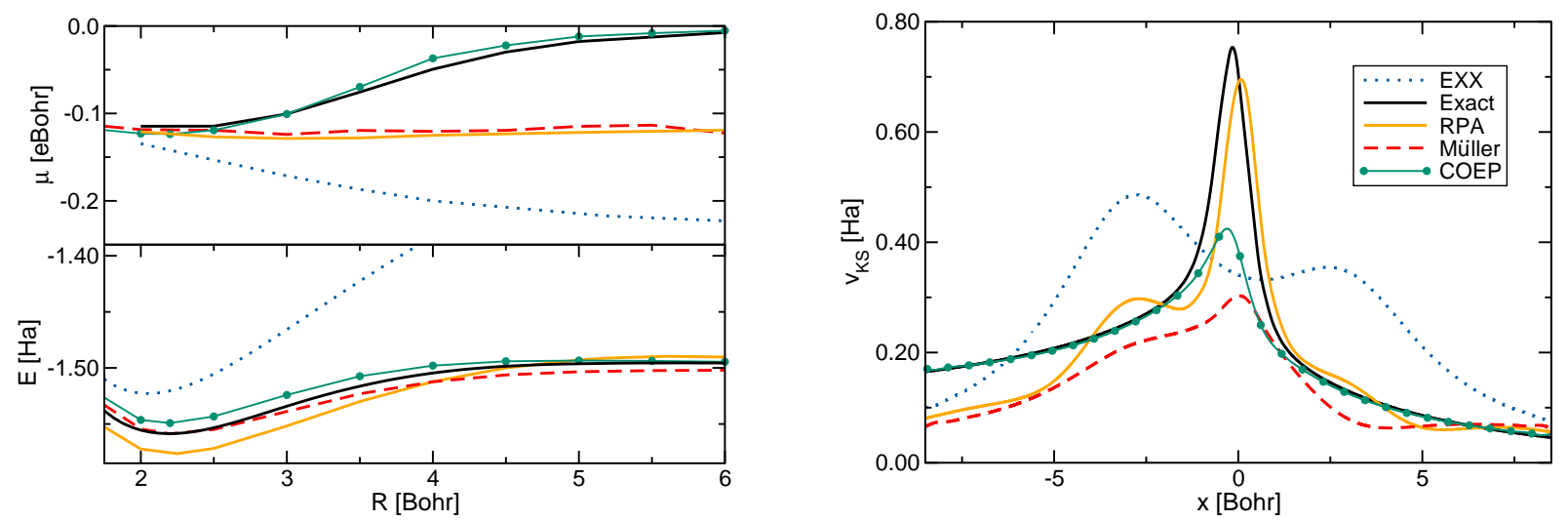

Figure 1: Left: The total energy $E$ and dipole moment $\mu$ (which highlights errors in the density) of the LiH molecule with EXX (blue dots), RPA (orange full), Müller (red dashed) and COEP (green filled circles) compared to exact results (black full). Right: The corresponding KS potentials at separation $R=6$ Bohr.

that an energy discontinuity is missing. Although simple improvements such as changing the square root of the occupation numbers to an optimised power improve energies ${ }^{49}$ fundamental issues remain. ${ }^{48}$

In the next section we will study how well the Müller functional performs in dissociating a heteronuclear dimer. We restrict the functional to HOMO/LUMO only, in order to maximize its similarity to COEP defined above. We will thus show that despite energies of similar quality to COEP, the delocalisation error remains as large as within a typical DFT approximation. We will further demonstrate that this error is related to a missing step of the Müller effective potential that is correctly captured by the COEP effective potential.

\section{Numerical analysis}

In this section we present a numerical analysis of a stretched heteronuclear dimer that mimics LiH-like molecules at dissociation. To this end, we introduce soft-coulomb interactions in one dimension. ${ }^{50-52}$ Thus, the electron-electron interaction is $v\left(x-x^{\prime}\right)=1 / \sqrt{1+\left(x-x^{\prime}\right)^{2}}$ 
and the external potential is

$$
V_{\mathrm{Ext}}=\frac{Z_{1}}{\sqrt{1+(x+R / 2)^{2}}}+\frac{Z_{2}}{\sqrt{1+(x-R / 2)^{2}}},
$$

where $Z_{1}=-1.2$ and $Z_{2}=-1$. The difference between the ionization energies of the two $1 \mathrm{D}$ atoms is $0.2 \mathrm{Ha}$, which can be compared to the $\mathrm{LiH}$ value of $0.3 \mathrm{Ha}$. Other choices of parameters are also possible. ${ }^{50,52}$ The core electrons on the $1 \mathrm{D}$ 'Li' atom are frozen and hence the ' $\mathrm{LiH}$ ' is a two electron system for which we have a numerically exact solution to compare with. Calculations are done in a 1D spline basis-set previously proven suitable to solve OEP-type of problems. ${ }^{40,41}$ LDA and exact results are obtained with the OCTOPUS code. ${ }^{51,53}$

All calculations are performed self-consistently. The potential for the COEP and Müller functionals are defined by applying Eq. (12) to Eq. (10) and Eq. (17), respectively. The random-phase approximation (RPA) potential is found via the linearized Sham-Schlüter equation as described in previous works. ${ }^{40,54-56}$

We will start by looking at the potential energy curve $E$ and dipole moment $\mu$ and then analyse the ability of approximate functionals to deal with charge localisation, and relate this ability to energy derivative discontinuities and step features in the Hxc potential.

\subsection{LiH dissociation}

In a stretched symmetric or homonuclear system like $\mathrm{H}_{2}$ the KS HOMO and LUMO are both delocalized by symmetry contraints. As a consequence, when $\theta=\pi / 4$ the correlated orbitals are completely localized, and, due to the infinitesimal overlap between $\psi_{A}$ and $\psi_{B}$, the interaction energy in Eq. (8) is close to zero. There is thus no static correlation error in COEP.

In the more challenging heteronuclear systems the KS orbitals may, or may not, be delocalized, depending crucially on the behavior of the KS potential. If the electrons 
were completely non-interacting the HOMO/LUMO would be localized on the atom with stronger/weaker nuclear potential. Hence, in the dissociation limit we would find both electrons on the atom with the largest ionization energy and zero on the other. In the $\mathrm{LiH}$ molecule the repulsive electron-electron interaction changes the charge distribution favouring one electron on each atom in the dissociation limit. This system thus provide a critical test of the quality of electron densities obtained via approximations, quantified through the quality of the dipole moment $\mu=\int\left[n(x)-n_{\text {nuclear }}(x)\right] x d x$.

Standard KS DFT, which relies on a doubly occupied HOMO, solves this problem by aligning the effective HOMO of the two atoms via a step in the KS potential when calculated exactly. ${ }^{12,57}$ In this way the orbitals delocalize and the doubly occupied HOMO simulates localized electrons. However, all known approximate functionals in DFT fail to achieve the step when open shell atoms are dissociated. Consequently, fractionally charged atoms are found in the dissociation limit together with a diverging dipole moment: $|\mu(R)| \rightarrow \infty$ for $R \rightarrow \infty$.

How functionals that exploit fractionally occupied KS orbitals deal with the same situation is presently not well understood. From our accurate COEP approximation (Eq. (8)) we see that the KS orbitals must delocalize to ensure the interaction energy vanishes in the dissociation limit. Therefore, we expect a step feature similar to the one in DFT to develop in the KS potential of Eq. (12).

In Fig. 1 (left) we show the potential energy curves and dipole moment of our model LiH molecule using the Müller, RPA, EXX and COEP functionals and compare them to the exact result. As for $\mathrm{H}_{2}$, we see that both Müller and COEP are close to free from static correlation errors and have similar overall performance on energies. Müller out-performs COEP near contact due to a better treatment of dynamical correlation. But it becomes worse during dissociation. RPA also performs well near contact and in the dissociation limit. Nonetheless, all approximations (except EXX) give satisfactory dissociation curves and total energies.

However, we see a drastically different behaviour for the dipole moment, $\mu$. The dipole 

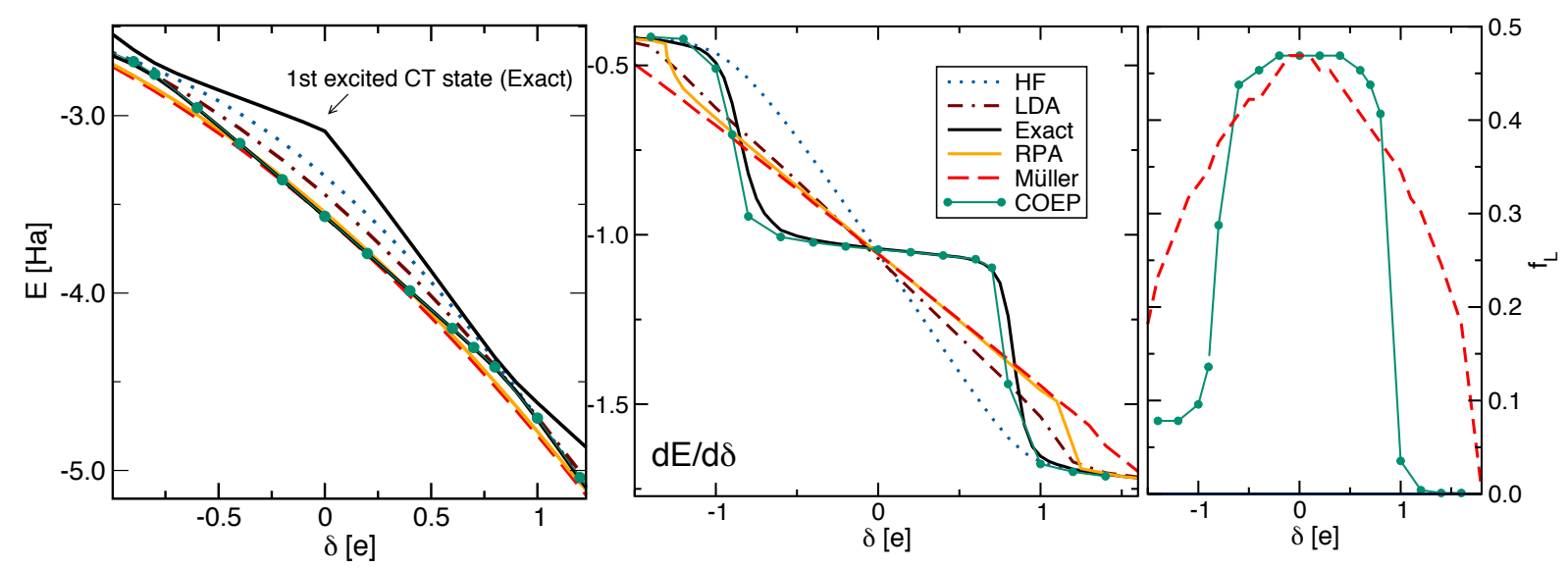

Figure 2: Left: Total energy as a function of the strength of the potential of atom $2\left(Z_{2}=\right.$ $-2-\delta$ ) with HF, LDA, RPA, Müller and COEP compared to exact result. Also the first excited singlet state is shown. Middle: Derivative of the ground state energy with respect to $\delta$ for the different approximations. Right: The corresponding occupation of the LUMO orbital within Müller and COEP.

moment serves as an indirect measure of the charge distribution, as discussed above. It has previously been used to assess the quality of densities from quantum chemical approximations. ${ }^{58}$ While COEP gives very accurate results, both Müller and RPA fail. A diverging dipole moment implies that the molecule dissociates into fractionally charged atoms. This problem, previously discussed for RPA, $, 11,59$ has so far not been recognized for functionals in RDMFT. Our work thus establishes that RDMFT is not a guaranteed solution for this problem as it is not free from the delocalization errors that plague DFT.

\subsection{The role of the potential}

By looking at the corresponding KS potentials (Fig. 1, right) the origin of the density error becomes clear. The exact KS potential of DFT (black full line) exhibits a peak at around the bond midpoint, and a step that aligns the ionization potentials of the atoms. The peak has its origin in kinetic correlation and acts to separate the charges. ${ }^{60,61}$ This peak is present also in the RPA but cannot alone prevent delocalisation. ${ }^{9,11}$ Due to the improved description of kinetic correlation in KS theories with fractional orbital occupations, like RDMFT, the peak is expected to be less relevant. This is, indeed, the case as demonstrated by the much 
smaller peak found in COEP.

We next turn our attention to the step. The step feature can be observed already in the potential of the EXX approximation when dissociating molecules into closed shell fragments ${ }^{32}$ or when applying an electric field to molecular chains. ${ }^{33,62,63}$ However, in the case of LiH-like dissociation none of EXX, RPA or the Müller approach exhibit this feature. In contrast, COEP exhibits a step of exactly the same size as the exact KS potential of DFT. The missing step in both Müller and RPA explains the error in the their densities; and hence the divergent dipole moment.

From this simple demonstration we can conclude that the step is not only an essential feature of the DFT KS potential but also of the local potential used in local RDMFT theory or in wavefunction/ensemble theories that exploit optimized KS orbitals. We emphasise that orbital optimisation in LiH-like molecules is crucial as the EXX orbitals are clearly unsuitable for either accurate energies or densities.

Finally, we note that our work closely parallels recent results from Oueis and Wassermann $^{52}$ in the context of exact partitioning theories. They studied the exact pinning potential of dissociated 1D $\mathrm{H}_{2}$ and $\mathrm{LiH}$ (using similar electronic models to those considered here). The pinning potential, which incorporates non-local quantum effects to supplement local models based on partitioning of the diatoms into atoms, was shown to have analogous step and peak features to those found here, which are caused by the same fundamental physics.

\subsection{Delocalisation error and derivative discontinuity}

We will now analyse in more detail the origin of the step in the KS potential. In DFT the step has been related to derivative discontinuities of the exact xc energy functional when

studied as a function of particle number. ${ }^{12,64}$ To expose these discontinuities an extension of DFT functionals to ensembles composed of states with different particle numbers is required. Most approximate functionals have, however, been shown to miss the derivative discontinuity leading to an incorrect dissociation limit with atomic densities integrating to a fractional 
number of particles. The delocalisation error is therefore sometimes called fractional charge error. $^{4}$

To continue our analysis, and uncover the properties of functionals involving fractionally occupied KS orbitals we will use an alternative approach, introduced in Ref. 65, which does not involve a direct treatment of ensembles. Specifically, we study a stretched molecule at large separation and vary the nuclear charge on one of the atoms to transform the properties of the groundstate system. In our model system we set $R=5$, set the charge on the first atom to $Z_{1}=-2$ and, set the second to $Z_{2}=-2-\delta$ where $\delta$ is varied between -1.5 and 1.5. We then calculate the total ground state energy as a function $\delta$. By varying $\delta$ we are able to change the number of electrons that localize on the two atoms. For certain values of $\delta$ (e.g. $\delta \approx-0.9)$, the number or left and right electrons can be varied continuously and the two atoms attain fractional charges, as detailed below. Since the well-separated atoms are only weakly coupled, they become closely analogous to isolated atoms attaining fractional charges via interactions with a bath.

In the exact calculation we have access to both ground and excited states. When $\delta<$ -0.9 the exact ground state $\left|\Psi_{0}\right\rangle$ has two electrons on atom 2 and none on atom 1 . The first singlet excited state $\left|\Psi_{1 S}\right\rangle$ corresponds to a charge transfer state where one electron on atom 2 is transferred to atom 1 (see, e.g., Refs. 50,66). At around $\delta=-0.9,\left|\Psi_{0}\right\rangle$ and $\left|\Psi_{1 S}\right\rangle$ become nearly degenerate (exactly so in the limit of infinite separation) and it is possible to form an arbitrary linear combination of them that has (almost) the same energy. These states are allowed to have fractional charges on the atoms even at infinite separation and hence the situation corresponds to the one of studying one atom in an ensemble of states. As soon as $\delta>-0.9$, the excited state with one electron on each atom (i.e. the first excited charge-transfer state) becomes the ground state and vice versa.

In Fig. 2 we plot the energy and its derivative with respect to $\delta$ as functions of $\delta$ for the exact case, COEP, Müller, EXX, LDA and RPA. In the energy plot we also include the energy of the first exact singlet excited state. The most notable feature is that, in the exact 
calculation, we see kinks or derivative discontinuities appearing at the "switching points" between the ground and the excited state. These discontinuities becomes sharper the further apart the atoms are.

Turning to the approximations, we see that EXX performs well until $\delta$ crosses the point where the ground- and excited state become degenerate in the exact calculation. As soon as the charge-transfer state should be favoured the EXX predicts a mixture of the two states leading to spurious fractional charges on the atoms. This error is consistent with the large delocalisation errors found at dissociation of $\mathrm{LiH}$ (see Fig. 1) and allows us to view the delocalisation (or fractional charge) error as an artificial mixing of the ground state with the first excited charge-transfer state beyond the point of degeneracy. A similar interpretation is often given to the fractional spin error but as an artificial mixing with the first triplet excited state (not shown in the figure as spin-symmetry is restricted here).

The connection between the derivative discontinuity in the energy and the step in potential has been made previously in the literature. ${ }^{12,64}$ In addition, it has been demonstrated that the development of the potential step during dissociation is connected to the appearance of an avoided crossing between the ground state and a charge-transfer excited state. ${ }^{50}$ Our analysis shows how these two pictures can be merged. We also note that when dissociating a strongly ionic bond as in $\mathrm{NaCl}$ the avoided crossing could become close to infinitely sharp, ${ }^{14}$ a situation more similar to the "switching point" in our model at large separation but, in this case, occurring already at finite separation.

The RPA clearly performs better than EXX. Nonetheless, the derivative discontinuities are missed, leading to delocalisation errors of similar magnitude to LDA. ${ }^{11}$ In view of the results in the previous section it is not surprising to see that the Müller functional fails as badly as RPA. Despite having an ostensibly good treatment of strong correlations, it lacks the ability to correctly treat charge localization.

Only the COEP, which is close to the exact functional in RDMFT, is able to reproduce the correct behaviour with an energy discontinuity, or a step in the derivative. We note that 
restricting to a local potential does not impose any severe restriction even in this challenging situation. This can thus not be blamed for the failure of the Müller functional, for example.

In the right panel of Fig. 2 we plot the occupation numbers in COEP and the Müller functional. While the occupation numbers of the Müller functional are smooth as a function of $\delta$, the occupation numbers of COEP exhibit discontinuities at the points where the energy has derivative discontinuities. Thus, just as in DFT, the problem of delocalization in RDMFT can be related to missing discontinuities in the energy functional. Fig. 2 (right) provides evidence that these discontinuities appear directly in the occupation numbers whenever charge localization is appropriately dealt with, as in COEP.

\section{Conclusions}

In this work we have analyzed the optimized effective potential within approaches that deal explicitly with fractional occupation numbers. In particular, we have studied the challenging case of heteronuclear dissociation and compared the potential to the KS potential of DFT, showing that, e.g., the step remains an essential feature. We have also introduced a generally applicable stringent test that highlights the ability of approximate functionals in dealing with charge localization.

We showed that RDMFT approximations such as the Müller functional, just like RPA, miss an important energy derivative discontinuity, and thus suffer from rather large delocalization errors that lead to errors in the dipole moment. Furthermore, by employing a multiplicative OEP in the nearly exact COEP, we showed that these errors are avoided thanks to the appearance of the step features in the effective potential.

We thus recommend further attention be placed on the quality of the potential in theories that exploit fractional orbital occupation numbers such as RDMFT and, more generally, in wavefunction or ensemble theories. Our work indicates that using optimized effective potentials can significanly improve on existing techniques, and lead to improvements in the 
current state-of-art in electronic structure theory.

Finally, we note that these results have some implications in the context of density-driven and functional-driven analysis ${ }^{67}$ of RDMFT. The Müller functional gives good energies of $\mathrm{LiH}$ dissociation, despite rather poor densities, implying small density-driven errors. This behaviour should be explored in future work.

\section{Acknowledgement}

M.H. acknowledges Dr. Florian G. Eich for valuable discussions on RDMFT.

\section{References}

(1) Yang, W.; Zhang, Y.; Ayers, P. W. Degenerate Ground States and a Fractional Number of Electrons in Density and Reduced Density Matrix Functional Theory. Phys. Rev. Lett. 2000, 84, 5172-5175.

(2) Mori-Sánchez, P.; Cohen, A. J.; Yang, W. Many-electron self-interaction error in approximate density functionals. J. Chem. Phys. 2006, 125, 201102.

(3) Cohen, A. J.; Mori-Sánchez, P.; Yang, W. Insights into Current Limitations of Density Functional Theory. Science 2008, 321, 792-794.

(4) Mori-Sánchez, P.; Cohen, A. J.; Yang, W. Discontinuous Nature of the ExchangeCorrelation Functional in Strongly Correlated Systems. Phys. Rev. Lett. 2009, 102, 066403.

(5) Kohn, W.; Sham, L. J. Self-Consistent Equations Including Exchange and Correlation Effects. Phys. Rev. 1965, 140, A1133-A1138.

(6) Perdew, J. P.; Burke, K.; Ernzerhof, M. Generalized gradient approximation made simple. Phys. Rev. Lett. 1996, 77, 3865-3868. 
(7) Becke, A. D. Density-functional exchange-energy approximation with correct asymptotic behavior. Phys. Rev. A 1988, 38, 3098-3100.

(8) Bruneval, F. GW Approximation of the Many-Body Problem and Changes in the Particle Number. Phys. Rev. Lett. 2009, 103, 176403.

(9) Hellgren, M.; Rohr, D. R.; Gross, E. K. U. Correlation potentials for molecular bond dissociation within the self-consistent random phase approximation. J. Chem. Phys. 2012, 136, 034106.

(10) Caruso, F.; Rohr, D. R.; Hellgren, M.; Ren, X.; Rinke, P.; Rubio, A.; Scheffler, M. Bond Breaking and Bond Formation: How Electron Correlation is Captured in ManyBody Perturbation Theory and Density-Functional Theory. Phys. Rev. Lett. 2013, 110, 146403.

(11) Colonna, N.; Hellgren, M.; de Gironcoli, S. Molecular bonding with the RPAx: From weak dispersion forces to strong correlation. Phys. Rev. B 2016, 93, 195108.

(12) Perdew, J. P.; Parr, R. G.; Levy, M.; Balduz, J. L. Density-Functional Theory for Fractional Particle Number: Derivative Discontinuities of the Energy. Phys. Rev. Lett. 1982, 49, 1691-1694.

(13) Makmal, A.; Kümmel, S.; Kronik, L. Dissociation of diatomic molecules and the exactexchange Kohn-Sham potential: The case of LiF. Phys. Rev. A 2011, 83, 062512.

(14) Ruzsinszky, A.; Perdew, J. P.; Csonka, G. I.; Vydrov, O. A.; Scuseria, G. E. Spurious fractional charge on dissociated atoms: Pervasive and resilient self-interaction error of common density functionals. J. Chem. Phys. 2006, 125, 194112.

(15) Vydrov, O. A.; Scuseria, G. E.; Perdew, J. P. Tests of functionals for systems with fractional electron number. J. Chem. Phys. 2007, 126, 154109. 
(16) Cohen, A. J.; Mori-Sánchez, P.; Yang, W. Development of exchange-correlation functionals with minimal many-electron self-interaction error. J. Chem. Phys. 2007, 126, 191109.

(17) Baer, R.; Livshits, E.; Salzner, U. Tuned Range-Separated Hybrids in Density Functional Theory. Annu. Rev. Phys. Chem. 2010, 61, 85-109, PMID: 20055678.

(18) Tsuneda, T.; Song, J.-W.; Suzuki, S.; Hirao, K. On Koopmans' theorem in density functional theory. J. Chem. Phys. 2010, 133, 174101.

(19) Johnson, E. R.; Contreras-García, J. Communication: A density functional with accurate fractional-charge and fractional-spin behaviour for s-electrons. J. Chem. Phys. 2011, 135, 081103.

(20) Kraisler, E.; Kronik, L. Piecewise Linearity of Approximate Density Functionals Revisited: Implications for Frontier Orbital Energies. Phys. Rev. Lett. 2013, 110, 126403.

(21) Steinmann, S. N.; Yang, W. Wave function methods for fractional electrons. J. Chem. Phys. 2013, 139, 074107.

(22) Zheng, X.; Zhou, T.; Yang, W. A nonempirical scaling correction approach for density functional methods involving substantial amount of Hartree-Fock exchange. J. Chem. Phys. 2013, 138, 174105.

(23) Malet, F.; Gori-Giorgi, P. Strong Correlation in Kohn-Sham Density Functional Theory. Phys. Rev. Lett. 2012, 109, 246402.

(24) Vuckovic, S.; Gori-Giorgi, P. Simple Fully Nonlocal Density Functionals for Electronic Repulsion Energy. J. Phys. Chem. Lett. 2017, 8, 2799-2805, PMID: 28581751.

(25) Löwdin, P.-O.; Shull, H. Natural Orbitals in the Quantum Theory of Two-Electron Systems. Phys. Rev. 1956, 101, 1730-1739. 
(26) Donnelly, R. A.; Parr, R. G. Elementary properties of an energy functional of the first-order reduced density matrix. J. Chem. Phys. 1978, 69, 4431-4439.

(27) Sharp, R. T.; Horton, G. K. A Variational Approach to the Unipotential Many-Electron Problem. Phys. Rev. 1953, 90, 317-317.

(28) Talman, J. D.; Shadwick, W. F. Optimized effective atomic central potential. Phys. Rev. A 1976, 14, 36-40.

(29) Schönhammer, K.; Gunnarsson, O. Discontinuity of the exchange-correlation potential in density functional theory. J. Phys. C: Solid State Phys. 1987, 20, 3675.

(30) Krieger, J. B.; Li, Y.; Iafrate, G. J. Construction and application of an accurate local spin-polarized Kohn-Sham potential with integer discontinuity: Exchange-only theory. Phys. Rev. A 1992, 45, 101-126.

(31) Gori-Giorgi, P.; Savin, A. Study of the discontinuity of the exchange-correlation potential in an exactly soluble case. Int. J. Quantum Chem. 2009, 109, 2410-2415.

(32) Hellgren, M.; Gross, E. K. U. Discontinuities of the exchange-correlation kernel and charge-transfer excitations in time-dependent density-functional theory. Phys. Rev. A 2012, 85, 022514 .

(33) Hellgren, M.; Gross, E. K. U. Discontinuous functional for linear-response timedependent density-functional theory: The exact-exchange kernel and approximate forms. Phys. Rev. A 2013, 88, 052507.

(34) Gidopoulos, N. I.; Papaconstantinou, P. G.; Gross, E. K. U. Spurious Interactions, and Their Correction, in the Ensemble-Kohn-Sham Scheme for Excited States. Phys. Rev. Lett. 2002, 88, 033003.

(35) Grabowski, I.; Hirata, S.; Ivanov, S.; Bartlett, R. J. Ab initio density functional theory: 
OEP-MBPT(2). A new orbital-dependent correlation functional. J. Chem. Phys. 2002, $116,4415-4425$.

(36) Lathiotakis, N. N.; Helbig, N.; Rubio, A.; Gidopoulos, N. I. Local reduced-densitymatrix-functional theory: Incorporating static correlation effects in Kohn-Sham equations. Phys. Rev. A 2014, 90, 032511.

(37) Muller, R. P.; Desjarlais, M. P. Optimized effective potential from a correlated wave function: Optimized effective potential-generalized valence bond (OEP-GVB). J. Chem. Phys. 2006, 125, 054101.

(38) Weimer, M.; Della Sala, F.; Görling, A. Multiconfiguration optimized effective potential method for a density-functional treatment of static correlation. J. Chem. Phys. 2008, 128, 144109.

(39) Goddard III, W. A.; Dunning Jr, T. H.; Hunt, W. J.; Hay, P. J. Generalized valence bond description of bonding in low-lying states of molecules. Acc. Chem. Res. 1973, 6, 368-376.

(40) Hellgren, M.; von Barth, U. Correlation potential in density functional theory at the GWA level: Spherical atoms. Phys. Rev. B 2007, 76, 075107.

(41) Hellgren, M.; von Barth, U. Exact-exchange kernel of time-dependent density functional theory: Frequency dependence and photoabsorption spectra of atoms. J. Chem. Phys. 2009, 131, 044110.

(42) Gross, E. K. U.; Oliveira, L. N.; Kohn, W. Rayleigh-Ritz variational principle for ensembles of fractionally occupied states. Phys. Rev. A 1988, 37, 2805-2808.

(43) Gross, E. K. U.; Oliveira, L. N.; Kohn, W. Density-functional theory for ensembles of fractionally occupied states. I. Basic formalism. Phys. Rev. A 1988, 37, 2809-2820. 
(44) Gould, T.; Pittalis, S. Hartree and Exchange in Ensemble Density Functional Theory: Avoiding the Nonuniqueness Disaster. Phys. Rev. Lett. 2017, 119, 243001.

(45) Gould, T.; Pittalis, S. Density-Driven Correlations in Many-Electron Ensembles: Theory and Application for Excited States. Phys. Rev. Lett. 2019, 123, 016401.

(46) Müller, A. M. K. Explicit Approximate Relation between Reduced Two- and OneParticle Density Matrices. Phys. Lett. A 1984, 105, 446.

(47) Buijse, M. A.; Baerends, E. J. An approximate exchange-correlation hole density as a functional of the natural orbitals. Mol. Phys. 2002, 100, 401.

(48) Kamil, E.; Schade, R.; Pruschke, T.; Blöchl, P. E. Reduced density-matrix functionals applied to the Hubbard dimer. Phys. Rev. B 2016, 93, 085141.

(49) Lathiotakis, N. N.; Sharma, S.; Dewhurst, J. K.; Eich, F. G.; Marques, M. A. L.; Gross, E. K. U. Density-matrix-power functional: Performance for finite systems and the homogeneous electron gas. Phys. Rev. A 2009, 79, 040501.

(50) Tempel, D. G.; Martínes, T. J.; Maitra, N. T. Revisiting Molecular Dissociation in Density FunctionalTheory: A Simple Model. J. Chem. Theory Comput. 2009, 5, 770780 .

(51) Helbig, N.; Fuks, J. I.; Casula, M.; Verstraete, M. J.; Marques, M. A. L.; Tokatly, I. V.; Rubio, A. Density functional theory beyond the linear regime: Validating an adiabatic local density approximation. Phys. Rev. A 2011, 83, 032503.

(52) Oueis, Y.; Wasserman, A. Exact partition potential for model systems of interacting electrons in 1-D. Euro. Phys. J. B 2018, 91, 247.

(53) Marques, M. A. L.; Castro, A.; Bertsch, G. F.; Rubio, A. octopus: a tool for the application of time-dependent density functional theory. Phys. Status Solidi B 2006, 243, 2465. 
(54) Sham, L. J.; Schlüter, M. Density-Functional Theory of the Energy Gap. Phys. Rev. Lett. 1983, 51, 1888-1891.

(55) Casida, M. E. Generalization of the optimized-effective-potential model to include electron correlation: A variational derivation of the Sham-Schlüter equation for the exact exchange-correlation potential. Phys. Rev. A 1995, 51, 2005-2013.

(56) von Barth, U.; Dahlen, N. E.; van Leeuwen, R.; Stefanucci, G. Conserving approximations in time-dependent density functional theory. Phys. Rev. B 2005, 72, 235109.

(57) Maitra, N. T. Undoing static correlation: Long-range charge transfer in time-dependent density-functional theory. J. Chem. Phys. 2005, 122, 234104.

(58) Hait, D.; Head-Gordon, M. How accurate is density functional theory at predicting dipole moments? an assessment using a new database of 200 benchmark values. Journal of chemical theory and computation 2018, 14, 1969-1981.

(59) Mori-Sánchez, P.; Cohen, A. J.; Yang, W. Failure of the random-phase-approximation correlation energy. Phys. Rev. A 2012, 85, 042507.

(60) Buijse, M. A.; Baerends, E. J.; Snijders, J. G. Analysis of correlation in terms of exact local potentials: Applications to two-electron systems. Phys. Rev. A 1989, 40, 41904202.

(61) Gritsenko, O. V.; Baerends, E. J. Effect of molecular dissociation on the exchangecorrelation Kohn-Sham potential. Phys. Rev. A 1996, 54, 1957-1972.

(62) van Gisbergen, S. J. A.; Schipper, P. R. T.; Gritsenko, O. V.; Baerends, E. J.; Snijders, J. G.; Champagne, B.; Kirtman, B. Electric Field Dependence of the ExchangeCorrelation Potential in Molecular Chains. Phys. Rev. Lett. 1999, 83, 694-697.

(63) Kümmel, S.; Kronik, L.; Perdew, J. P. Electrical Response of Molecular Chains from Density Functional Theory. Phys. Rev. Lett. 2004, 93, 213002. 
(64) Hodgson, M. J. P.; Kraisler, E.; Schild, A.; Gross, E. K. U. How Interatomic Steps in the Exact Kohn-Sham Potential Relate to Derivative Discontinuities of the Energy. $J$. Phys. Chem. Lett. 2017, 8, 5974.

(65) Cohen, A. J.; Mori-Sánchez, P. Dramatic changes in electronic structure revealed by fractionally charged nuclei. J. Chem. Phys. 2014, 140, 044110.

(66) Gould, T.; Kronik, L.; Pittalis, S. Charge transfer excitations from exact and approximate ensemble Kohn-Sham theory. J. Chem. Phys. 2018, 148, 174101.

(67) Kim, M.-C.; Sim, E.; Burke, K. Understanding and Reducing Errors in Density Functional Calculations. Phys. Rev. Lett. 2013, 111, 073003. 
Figure 3: For Table of Contents Only

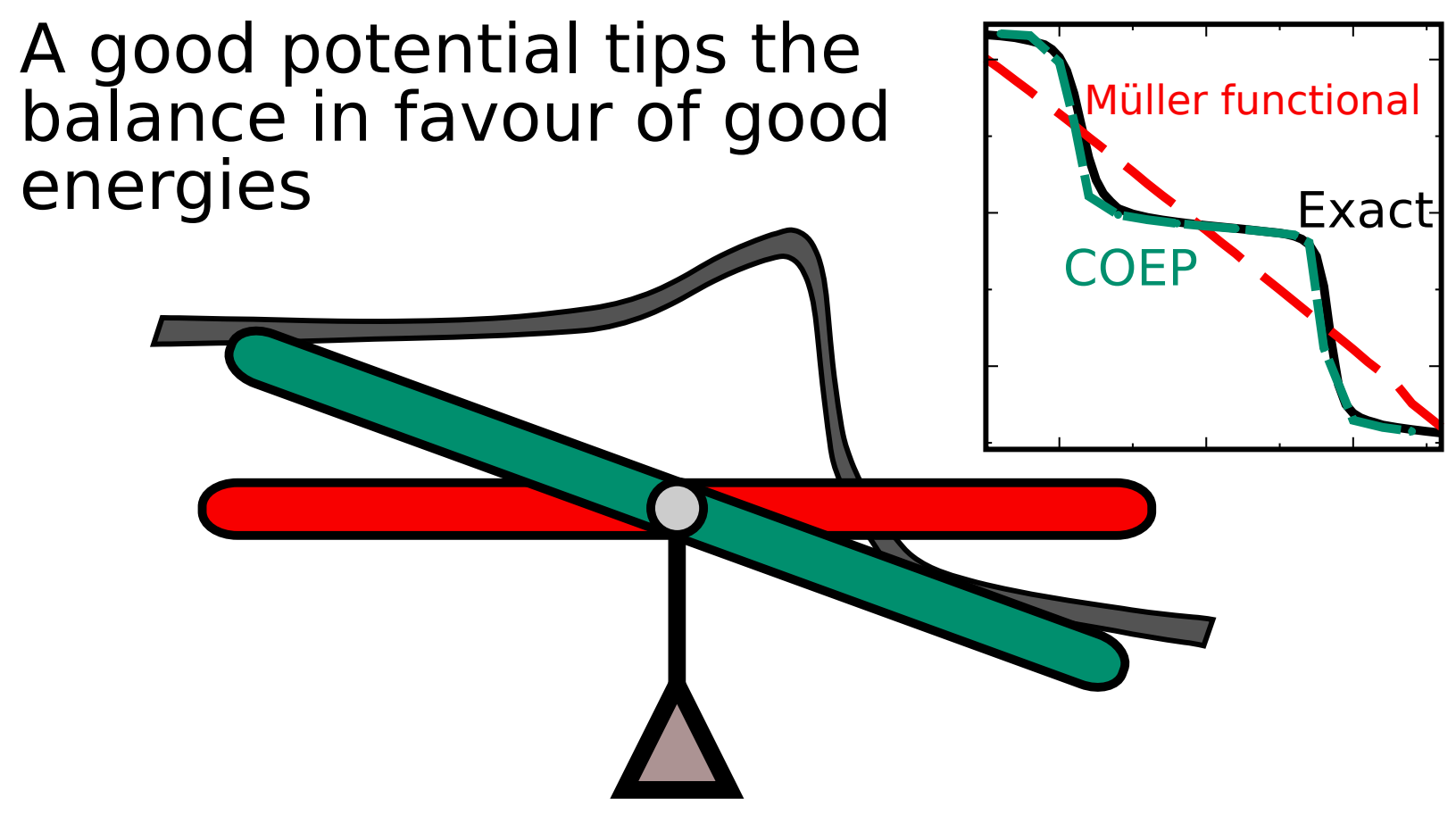

